\title{
Wavelet-Based Post-Processing Methods for the Enhancement of Non-Invasive Fetal ECG
}

\author{
Giulia Baldazzi ${ }^{1,2}$, Eleonora Sulas ${ }^{2}$, Elisa Brungiu ${ }^{2}$, Monica Urru ${ }^{3}$, \\ Roberto Tumbarello ${ }^{3}$, Luigi Raffo ${ }^{2}$, Danilo Pani ${ }^{2}$ \\ ${ }^{1}$ DIBRIS, University of Genova, Genova, Italy \\ ${ }^{2}$ Department of Electrical and Electronic Engineering, University of Cagliari, Cagliari, Italy \\ ${ }^{3}$ Division of Pediatric Cardiology, San Michele Hospital, Cagliari, Italy
}

\begin{abstract}
Despite the number of techniques developed in the literature, the extraction of a clean fetal ECG (fECG) from non-invasive recordings is still an open research issue. In this work, different wavelet-based post-processing approaches for the denoising of the fECG were evaluated. A small dataset composed of twenty signals recorded from ten pregnant women between the 21st and the 27th week of gestation was adopted. fECG extraction was accomplished by using a multireference QR-decomposition-based recursive least squares adaptive filter. Then, all signals were decomposed with the stationary wavelet transform (SWT) and stationary wavelet packet transform (SWPT), using a 7-level decomposition with Haar mother wavelet and hard-thresholding. Two different thresholds from the literature were tested: the first one is level-independent (Minimax) while the other one is level-dependent. The latter was adapted to be exploited on SWPT. The enhancement of the fetal QRS complex was analyzed by computing the improvement of the signal-to-noise ratio and the performance of a fetal QRS detector. The comparative analysis revealed how the SWT outperforms the more complex SWPT, regardless the thresholding approach.
\end{abstract}

\section{Introduction}

The antenatal screening has a primary role considering the incidence of congenital heart diseases. The ultrasound methods represent the usual care, whereas the adoption of the non-invasive fetal ECG (fECG) is still largely relegated to the heart rate analysis. Among the different reasons behind this, the relatively low signal-to-noise ratio (SNR) has a big impact [1]. The powerline and instrumental noises, the electrophysiological maternal interference, and its spectral overlap with the fECG, the attenuation of the signal due to the different layers of tissue to be passed through for gathering the signal on the maternal skin, cause the very low SNR of the fECG.

Beyond relying on an optimal acquisition setup, SNR can be enhanced by improving the three main steps of the
fECG extraction process: pre-processing, separation and post-processing. Despite the large number of works on the separation step, few works focused on the post-processing. The oldest and most widely used technique is the averaging of the consecutive ECG complexes [2], [3]. In [4], the time-sequenced adaptive filter was applied to enhance the quality of multichannel fECG after the maternal ECG had been removed. In [5], the enhancements achievable by the application of wavelet transform to fECG signals extracted by polynomial networks were presented.

In this work, different wavelet denoising postprocessing methods for the enhancement of non-invasive fECG were studied. Real non-invasive recordings from ten pregnant women were processed by multi-reference adaptive filtering, and the obtained fECG traces were decomposed with the stationary wavelet transform (SWT) and the stationary wavelet packet transform (SWPT). A 7level decomposition with Haar mother wavelet was used, along with hard thresholding, with two different thresholds: the Minimax and a level-dependent one by Han et al. [6]. Since their definitions were conceived for the standard wavelet decomposition, in this work they were opportunely adapted for the SWPT decomposition. This investigation was meant to perform a targeted denoising based on the fECG spectral bands of major interest, with coarser (SWT) or finer (SWPT) granularity.

\section{Materials and Methods}

Twenty multichannel non-invasive recordings from ten voluntary pregnant women between the 21 st and the 27 th weeks of gestation, with healthy fetuses, were recorded with the Porti7 portable physiological measurement system (TMSi, The Netherlands) at a sampling frequency of $2048 \mathrm{~Hz}$. For each recording, three non-coplanar bipolar thoracic leads were measured, and four among the twenty-four unipolar abdominal leads were used to give rise to two bipolar leads by digital subtraction. Simultaneously, the cardiac pulsed-wave Doppler signal was acquired by a Philips iE33 Ultrasound Machine (Philips, The Netherlands) in order to provide a ground truth to confirm the presence of fetal QRS complexes. 
Recordings were performed at the Division of Pediatric Cardiology of the S. Michele Hospital (Cagliari, Italy). The study was approved by the Independent Ethical Committee of the Cagliari University Hospital (AOU Cagliari) and followed the principles outlined in the Helsinki Declaration of 1975. All the women provided their signed informed consent to the protocol.

The raw signals were pre-processed in order to remove the baseline wandering artefacts by high-pass filtering at $1 \mathrm{~Hz}$ (1124-th order linear-phase equiripple finite impulse response filter). Then, exploiting the three ECG thoracic leads, the maternal ECG components were removed from the abdominal leads by a multi-reference QR-decomposition-based recursive least squares (QRDRLS) adaptive filter, with the forgetting factor set to 0.999 and the length of the filter to 20 [7]. Finally, 20 processed abdominal leads (10 seconds long each) were extracted to produce the testing dataset used in this work.

\subsection{Wavelet denoising}

Wavelet denoising is a powerful signal processing technique widely exploited in biomedical field. It is based on three different main steps [8]:

1. Wavelet transform by dyadic decomposition of the noisy signal in the time-scale domain (analysis).

At this stage, the noisy signal is given as input to a couple of high-pass and low-pass filters, whose kernels are defined on the basis of the selected mother wavelet, in charge to split in two equal parts the input signal band. In the classical wavelet transform, the same procedure is iteratively applied to the output of the low-pass filter for a total number of times equal to the chosen decomposition level $l$. The filter outputs related to the lowest sub-band are called approximation coefficients whereas the others, whose spectral bands span from folding frequency $f_{n}$ down to $f_{n} / 2^{l}$, are named details.

In a different approach, known as wavelet packet, the outputs of the high-pass filters are also decomposed, resulting in a complete binary tree with $2^{l}$ nodes or leaves, thus achieving a finer sub-band division.

2. Thresholding of wavelet details.

In this phase, details are compared to a threshold, whose definition can be level-dependent or not. Coefficients below this threshold are set to zero while those above threshold can be differently manipulated according to the chosen algorithm: in case of soft thresholding, their values are rescaled by subtracting the threshold, whereas in case of hard thresholding, their values are preserved [9].

3. Reconstruction of the signal in time domain (synthesis).

Finally, thresholded details and approximation coefficients are given as input to a mirrored trellis of highpass and low-pass filters, whose definition depends on the wavelet properties (for orthogonal wavelet, quadrature mirror filters can be used, i.e. the filters in the synthesis trellis are the mirrored versions of the homologous ones in the analysis trellis, whereas biorthogonal wavelets make use of completely different filters for analysis and synthesis).

In this work, we chose the stationary wavelet transform (SWT) and the stationary wavelet packet transform (SWPT) because of their translation-invariant characteristic. In this way, the morphology of the denoised signals is not affected by the fetal QRS occurrence over time. A level decomposition equal to 7 was chosen, so that wavelet denoising spreads until the frequency components of major interest for fetal QRS complex (around 8-20 Hz). Moreover, Haar mother wavelet and hard thresholding were chosen for every denoising approach: the former because of its better performance compared to other mother wavelets (data not shown), the latter in order to avoid the shrinkage effect associated to soft-thresholding.

In order to compute the threshold value, the Minimax [9] and a level-dependent method by Han et al. [6] were adopted. In particular, the latter was conceived to be progressively more aggressive at the higher frequencies. Their definitions are, respectively:

$$
\begin{aligned}
& \theta_{\mathrm{j}}=\sigma_{\mathrm{j}}\left(0.3936+0.1829 \log _{2}(\mathrm{~N})\right) \\
& \theta_{j}=\left\{\begin{array}{cc}
\sigma_{j} \sqrt{2 \ln (\mathrm{N})} & \mathrm{j}=1 \\
\sigma_{\mathrm{j}} \sqrt{2 \ln (\mathrm{N})} / \ln (\mathrm{j}+1) & 1<\mathrm{j}<\mathrm{L} \\
\sigma_{\mathrm{j}} \sqrt{2 \ln (\mathrm{N})} / \sqrt{\mathrm{j}} & \mathrm{j}=\mathrm{L}
\end{array}\right.
\end{aligned}
$$

where $\mathrm{N}$ is the signal length in samples, $\mathrm{L}$ is the chosen decomposition level and $\sigma_{j}$ is the standard deviation of the noise. In particular, $\sigma_{j}$ was computed on a noisy portion of the signals in between fetal QRSs and estimated at each level $\mathrm{j}$ as:

$$
\sigma_{\mathrm{j}}=\frac{\mathrm{MAD}\left(\mathrm{cD}_{\mathrm{j}}\right)}{0.6745}
$$

being $\mathrm{cD}_{\mathrm{j}}$ the detail coefficients at the $\mathrm{j}$-th level and MAD the median absolute deviation of such coefficients.

Since these definitions fitted the SWT denoising approaches, in the case of the SWPT-based ones, thresholds were adapted. In particular, $\sigma_{j}$ was calculated at each leaf as in (3). However, for the level-dependent threshold proposed in [6], it was adjusted considering for each SWPT leaf the corresponding SWT detail level on the basis of its spectral range and then adopting the related definition as in (2). 


\subsection{Comparative analysis and metrics}

In order to assess wavelet denoising performance on the real dataset, three different quantitative measures were employed. The first one is the SNR, which was evaluated as:

$$
\mathrm{SNR}_{\mathrm{dB}}=20 \log _{10}\left(\frac{\mathrm{App}_{\mathrm{f}}}{4 \sigma}\right)
$$

where $\sigma$ is the standard deviation of the noise and $\mathrm{App}_{\mathrm{f}}$ represents the peak-to-peak amplitude of the average fetal QRS complex. The average QRS complex was calculated only on highly correlated fetal beats, considering a Pearson's correlation coefficient value higher than 0.6. A $40 \mathrm{~ms}$ window was supposed to entirely enclose a fetal QRS complex.

Then we computed the accuracy and the true positive rate of a fetal QRS detector [10], to investigate how the improvement of the denoised traces can turn out in a higher detection accuracy. Accuracy (Acc) and the true positive rate (TPR, or Sensitivity) were estimated as:

$$
\begin{gathered}
\text { Acc }=\frac{\mathrm{TP}}{\mathrm{TP}+\mathrm{FP}+\mathrm{FN}} \times 100 \\
\mathrm{TPR}=\frac{\mathrm{TP}}{\mathrm{TP}+\mathrm{FN}} \times 100
\end{gathered}
$$

where false positives (FP) and true positives (TP), as well as false negatives (FN), were exactly identified thanks to the fetal QRS annotations estimated by using the simultaneous Doppler acquisition.

All these comparative metrics were computed before and after wavelet-based post-processing. Statistical significance $(p<0.05)$ was assessed by using the Wilcoxon test, due to the non-normality of the distributions.

\section{Results}

The box-and-whisker plots representing the distributions of SNR, Accuracy and TPR over the acquired dataset are depicted in Figure 1.

It is evident that wavelet denoising improved the quality of the fECG signals, with a statistically significant median SNR improvement over $9 \mathrm{~dB}$, compared to the original traces $(p=0.0001$ for all the four cases). From this perspective, SWT and SWPT showed approximately an equivalent performance. However, the two thresholds presented an opposite behavior, since Han et al. [6] led to better performance on SWPT (median of $10.6 \mathrm{~dB}$ versus $9.1 \mathrm{~dB}$ achieved with SWT, $\mathrm{p}>0.05$ ) whereas Minimax presented better performance on SWT (median value of $11.0 \mathrm{~dB}$ versus $10.4 \mathrm{~dB}$ for SWPT, $\mathrm{p}>0.05$ ).

As can be also seen from Figure 1, wavelet denoising effect on fetal QRS detection accuracy is controversial. In fact, it seemed to improve the accuracy only for SWT with Han et al. threshold, but there is no statistical significance.

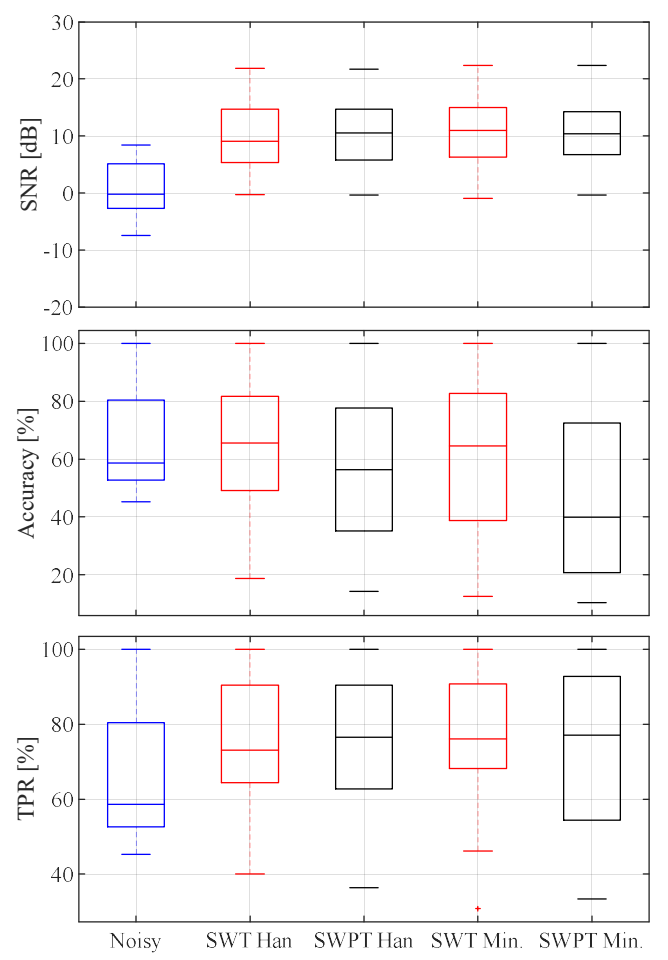

Figure 1. SNR (top), Acc (middle) and TPR (bottom) results obtained with SWT (red) and SWPT (black) denoising approaches. In each case, values are reported for noisy traces (blue) and for denoised ones, using the level-dependent threshold (by Han et al.) and the Minimax. Results were obtained on 20 traces from ten voluntary pregnant women with healthy fetuses.

The other improvements were associated to a wider distribution dispersion, so that the minimum accuracy was worse than that achievable without any post-processing. However, for SWT approaches, median values were always higher than initial ones $(64.57 \%$ for Minimax, $65.6 \%$ for Han et al. vs. $54.2 \%$ for noisy traces, $\mathrm{p}>0.05$ ). The same did not hold for SWPT.

Moreover, considering the same threshold, Acc performances were always significantly better in SWT implementation than SWPT one $(\mathrm{p}=0.01)$.

TPR results were significantly higher in post-processed signals compared to the original ones $(p<0.03$ for all the four cases). Focusing on the thresholds, TPRs were slightly higher in the cases of SWPT with respect to the SWT $(p>0.05)$. By looking at Acc and TPR, it is possible to guess that SWPT performance was affected by a larger number of FPs compared to SWT, which could be due to the stronger shrinkage effect of SWPT. This is confirmed by Figure 2, showing an example of denoised fECG traces. In general, SWT led to a better morphological preservation of the fECG signal whereas the SWPT led to lower fECG amplitude and significant distortions. In particular, the 


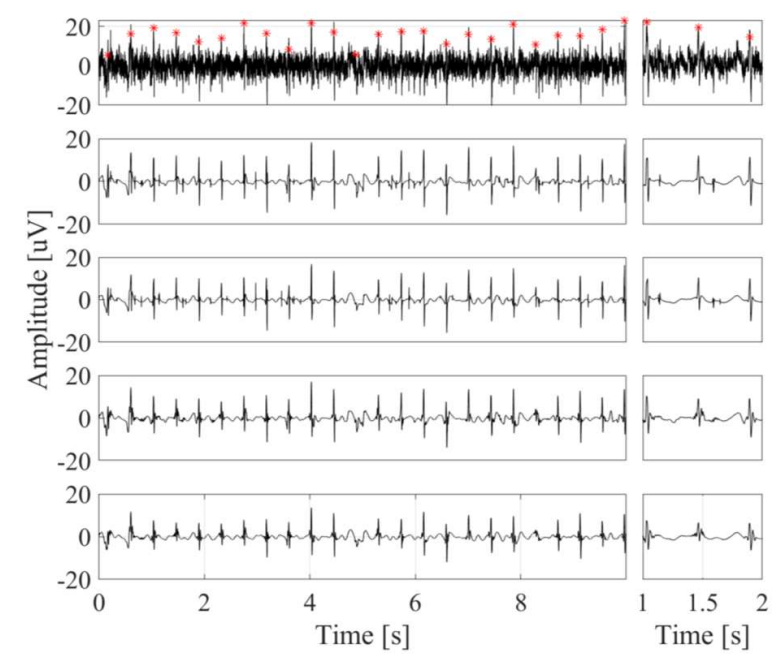

Figure 2. Example of SWT and SWPT denoising results with 7-level decomposition. From top to bottom: raw abdominal signal, SWT (Han et al.), SWT (Minimax), SWPT (Han et al.), SWPT (Minimax). On the right, a one-second zoom of each signal.

combination of the Minimax threshold and the SWPT induced larger distortions. Furthermore, the higher complexity of the SWPT should be compensated by a significantly better performance, which was not verified in the proposed assessment.

\section{Conclusions}

The post-processing enhancement of the fECG obtained from non-invasive recording, employing the SWT and the SWPT, was analyzed in terms of SNR, fetal QRS detection accuracy and TPR. On the available dataset, with the proposed processing scheme, featuring a 7-level decomposition with Haar mother wavelet and hardthresholding, the SWT outperformed the more complex SWPT in the Acc outputs, regardless the adopted threshold, but the Acc improvements with respect to the absence of denoising was negligible. QRS detection seems to be negatively affected by a high number of FPs in WD processed signals, which deserves further investigations.

Nevertheless, the results should be carefully analyzed in the light of some final considerations. At first, the SNR was computed on the average fetal QRS, which could lead to an underestimation because of the signal smoothing. The QRS detection accuracy and TPR are influenced by the chosen method, even though a robust technique was selected. Finally, SWPT thresholding was adapted exploiting a simple scheme, which could have had a negative impact on the measured performance. A more detailed study on this is then required to evaluate the best threshold adaptation strategy for SWPT in this specific scenario.

\section{Acknowledgments}

E. Sulas and G. Baldazzi contributed equally to this work. The authors wish to thank the team of the Division of Pediatric Cardiology, S. Michele Hospital (Cagliari, Italy), for the important support, and all the voluntary pregnant women. E. Sulas is grateful to Sardinia Regional Government for supporting her PhD scholarship (P.O.R. F.S.E., European Social Fund 2014-2020).

\section{References}

[1] M. T. Donofrio et al., "Diagnosis and treatment of fetal cardiac disease," Circulation, vol. 129, no. 21, pp. 21832242, May 2014.

[2] E. H. Hon, "Noise reduction in fetal electrocardiography.," Med. Arts Sci., vol. 18, pp. 63-66, 1964.

[3] X. B. Huang, J. A. Crowe, J. M. Herbert, and M. S. Woolfson, "A windows application for real-time fetal ECG analysis.," Comput. Biomed. Res., vol. 27, no. 6, pp. 419433, Dec. 1994.

[4] E. Fotiadou, J. O. E. H. van Laar, S. G. Oei, and R. Vullings, "Enhancement of low-quality fetal electrocardiogram based on time-sequenced adaptive filtering.," Med. Biol. Eng. Comput., vol. 56, no. 12, pp. 2313-2323, Dec. 2018.

[5] M. Ahmadi, M. Ayat, K. Assaleh, and H. Al-Nashash, "Fetal ECG signal enhancement using polynomial classifiers and wavelet denoising," in 2008 Cairo International Biomedical Engineering Conference, 2008, pp. 1-4.

[6] M. Han, Y. Liu, J. Xi, and W. Guo, "Noise smoothing for nonlinear time series using wavelet soft threshold," Signal Process. Lett. IEEE, vol. 14, pp. 62-65, 2007.

[7] E. Sulas, M. Urru, R. Tumbarello, L. Raffo, and D. Pani, "Comparison of single and multi-reference QRD-RLS adaptive filter for non-invasive fetal electrocardiography," in EMBC, 2019.

[8] M. Misiti, Y. Misiti, G. Oppenheim, and J.-M. Poggi, Wavelets and their applications, vol. 330. Wiley Online Library, 2007.

[9] D. L. Donoho and J. M. Johnstone, "Ideal spatial adaptation by wavelet shrinkage," Biometrika, vol. 81, no. 3, pp. 425455, 1994.

[10] J. Behar, J. Oster, and G. D. Clifford, "Combining and benchmarking methods of foetal ECG extraction without maternal or scalp electrode data," Physiol. Meas., vol. 35, no. 8, pp. 1569-1589, 2014.

Address for correspondence:

Giulia Baldazzi

Full postal address: P.zza d'Armi, 09123 Cagliari, Italy.

E-mail address: giulia.baldazzi@unica.it 